\title{
Fetal Heart Rate Monitoring from Phonocardiograph Signal Using Repetition Frequency of Heart Sounds
}

\author{
Hong Tang, ${ }^{1}$ Ting Li, ${ }^{2}$ Tianshuang Qiu, ${ }^{1}$ and Yongwan Park ${ }^{3}$ \\ ${ }^{1}$ Department of Biomedical Engineering, Dalian University of Technology, Dalian, China \\ ${ }^{2}$ College of Information and Communication Engineering, Dalian Minzu University, Dalian, China \\ ${ }^{3}$ Department of Information and Communication Engineering, Yeungnam University, Gyeongsan, Republic of Korea
}

Correspondence should be addressed to Hong Tang; tanghong@dlut.edu.cn

Received 1 July 2016; Revised 25 November 2016; Accepted 6 December 2016

Academic Editor: Jit S. Mandeep

Copyright (C) 2016 Hong Tang et al. This is an open access article distributed under the Creative Commons Attribution License, which permits unrestricted use, distribution, and reproduction in any medium, provided the original work is properly cited.

\begin{abstract}
As a passive, harmless, and low-cost diagnosis tool, fetal heart rate (FHR) monitoring based on fetal phonocardiography (fPCG) signal is alternative to ultrasonographic cardiotocography. Previous fPCG-based methods commonly relied on the time difference of detected heart sound bursts. However, the performance is unavoidable to degrade due to missed heart sounds in very low signal-to-noise ratio environments. This paper proposes a FHR monitoring method using repetition frequency of heart sounds. The proposed method can track time-varying heart rate without both heart sound burst identification and denoising. The average accuracy rate comparison to benchmark is $88.3 \%$ as the SNR ranges from $-4.4 \mathrm{~dB}$ to $-26.7 \mathrm{~dB}$.
\end{abstract}

\section{Introduction}

As a well-being policy, pregnant women in many countries are periodically demanded to monitor the variations in fetal heart rate (FHR). This monitoring of FHR and its variability provide up-to-date information about the fetus to prevent intrauterine death or permanent damage to the fetus [1]. Many techniques can monitor fetal heart rate previously. Fetal magnetocardiography (fMCG) using superconducting quantum interference device allows accurate assessment of beatto-beat fetal heart rate variability [2]. However, the equipment of fMCG is too expensive to be widely accessed in primary hospital. Cardiotocography (CTG) is continuous monitoring of the fetal heart rate using an ultrasound transducer placed on the mother's abdomen [3-5]. Physicians evaluate specific clinical CTG parameters by means of visual inspection. Hence, the accuracy of CTG monitoring is generally depending on observer's expertise. Fetal electrocardiogram (fECG) signal taken from the abdominal electrocardiogram of pregnant woman is another diagnostic tool for evaluating FHR [6]. However, the fECG signal is often masked by maternal ECG, power line interference, maternal electromyogram, and so forth. Very complex algorithms are needed to separate the fECG signal from mixed signal before further analysis.
As a totally passive methodology, fetal phonocardiography (fPCG) signal collected from mother's abdomen using a microphone transducer was proposed about ten years ago to monitor FHR [7]. This new technique makes long-term and frequent measurements of FHR possible. They detected the burst shaped heart sounds from signal envelope and estimated FHR via the time difference of heart sound occurrence [8-16]. Consequently, the accuracy of FHR estimation relies on heart sound detection rate. The performance degrades greatly if heart sounds are missed and/or wrong heart sounds are detected. Denoising technique is usually used as preprocessing due to the low energy of fetal heart sounds and multiple interferences [17].

It is known that fetal heart sounds are originated from interaction between fetal heart and blood flow therein. The sounds are repetitive from one cardiac cycle to another. FHR monitoring is thus equal to tracking the local repetition frequency of heart sound in adjacent cardiac cycles. Hence, heart beat identification and denoising are then not necessary.

\section{Methodology}

2.1. Repetition Frequency Estimated by Cyclic Frequency Spectrum. In the field of signal processing theory, repetition 
frequency is usually characterized by cycle frequency. It means occurrence rate of a repetitive event in a period of time. For example, the cycle frequency of fetal heart sounds is $2.5 \mathrm{~Hz}$ if they repeat every 0.4 seconds. It is assumed that $x(t)$ is a virtual digital sequence of an fPCG signal with an exact cycle period $T$. The objective of FHR estimation is to extract the cycle frequency of the fetal heart sound. The time-varying autocorrelation is written as

$$
\begin{aligned}
R_{x}(t, \tau) \triangleq & \lim _{N \rightarrow \infty} \frac{1}{2 N+1} \\
& \cdot \sum_{n=-N}^{N} x\left(t+\frac{\tau}{2}+n T\right) x^{*}\left(t-\frac{\tau}{2}+n T\right),
\end{aligned}
$$

where $n$ is cycle index, $T$ is a real number denoting cycle period, $\tau$ is time delay, and $N$ determines the number of cycles involved in analysis. $R_{x}(t, \tau)$ must be periodic because fetal heart sounds are exactly cyclic. It can be expanded into Fourier series:

$$
R_{x}(t, \tau)=\sum_{\alpha=-\infty}^{+\infty} R_{x}(\alpha, \tau) e^{j 2 \pi \alpha t}
$$

where $\alpha$ is a real number called cycle frequency. The coefficient of the Fourier series can be calculated by the following:

$$
R_{x}(\alpha, \tau)=\left\langle x\left(t+\frac{\tau}{2}\right) x\left(t-\frac{\tau}{2}\right) e^{j 2 \pi \alpha t}\right\rangle_{t} .
$$

The operator $\langle\cdot\rangle_{t}$ means the time average. $R_{x}(\alpha, \tau)$ is called cyclic correlation function. It can be seen that it reduces to the traditional correlation if the cycle frequency $\alpha$ is fixed to zero. $R_{x}(\alpha, \tau)$ can be transformed into frequency domain via Fourier transform:

$$
S_{x}(\alpha, f)=\int_{-\infty}^{\infty} R_{x}(\alpha, \tau) e^{-j 2 \pi f \tau} d \tau
$$

$S_{x}(\alpha, f)$ is called cyclic spectral density. The physical meaning of $S_{x}(\alpha, f)$ indicates that $S_{x}(\alpha, f) \neq 0$ if the signal $x(t)$ has any cyclic component at cycle frequency $\alpha$. In the point of view of fPCG signal, $S_{x}(\alpha, f)$ must have a peak at heart rate because the heart sounds are dominant cyclic components at the heart rate in adjacent fetal cardiac cycles. Therefore, detecting FHR is equal to detecting the basic cycle frequency of the digital sequence. The frequency spectrum is not of interest in this paper. An integral is performed to $S_{x}(\alpha, f)$ to remove the frequency variable $f$ for the purpose of simplification:

$$
\gamma_{x}(\alpha)=\int_{\infty}^{\infty}\left|S_{x}(\alpha, f)\right| d f
$$

This is called cyclic frequency spectrum (CFS). Therefore, the FHR is indicated by the dominant peak of $\gamma_{x}(\alpha)$ :

$$
\mathrm{Hr}=\arg \left\{\max \left(\gamma_{x}(\alpha)\right)\right\} \times 60,
$$

where $\arg \{\cdot\}$ is the argument that meets the condition in the brace and $\max (\cdot)$ is the maximum operator. A sharp and outstanding peak means a high degree of repetition of heart sounds in the signal. The normal FHR is commonly in [110160] bpm. That is, the cycle frequency in consideration is in [1.82.7] Hz. For extreme value, the signals in this paper are analyzed in a greater range, $[1.33 .5] \mathrm{Hz}$. These extreme heart rates are $80 \mathrm{bpm}$ and $210 \mathrm{bpm}$, which correspond to bradycardia and tachycardia, respectively.

To test the performance of CFS, the well-known simulated fPCG signals proposed by Cesarelli et al. $[18,19]$ are used. These simulated signals considered different fetal physiological and pathological conditions and recording situations by simulation technique. The noises considered consist of vibrations created by maternal body organs, fetal movements, and unwanted sounds from surrounding environment. These simulated signals have been openly published in PhysioNet for freely public access and have been widely accepted for algorithm evaluation. For example, two segments of a simulated fPCG signal both have $6 \mathrm{~s}$ with sampling frequency of $1 \mathrm{KHz}$, where the true real-time heart rate is between $140 \mathrm{bpm}$ and $155 \mathrm{bpm}$, seen in Figure 1. There are noises which saturate the signal due to maternal fast body movements, acoustic sensor displacements, and/or high magnitude external noises. CFS analysis is applied to the signal. Figure 1(a) shows the low noise part of the fPCG signal in time domain. Figure 1(b) shows the CFS of the signal. It can be found that the dominant peak occurs at the cycle frequency of $2.35 \mathrm{~Hz}$; that is, the FHR of this segment is $2.35 * 60=141 \mathrm{bpm}$. Figure 1(c) shows the heavy noise part of the signal. The signal is so heavily contaminated by noises that the heart beats cannot be identified by human visual inspection. Figure 1(d) shows that the CFS still has an outstanding prominent peak as if the noise has no effect on the peak. The peak is located at $2.51 \mathrm{~Hz}(151 \mathrm{bpm})$. The example illustrates that CFS can indicate the repetition frequency of the heart sounds in this segment. CFS analysis takes the segment as an integral input. Hence, heart sound detection that is essential in previous methods becomes not necessary in the proposed method. The noise is generally random and does not have any repetition feature. So the dominant peak of CFS will not be affected even the noise is so heavy to saturate the amplitude, as the example given in Figure 1(c).

It should be noted that the heart sounds are not perfectly periodic due to the heart rate variability (HRV). So, the heart sounds are quasi-periodic. The degree of the periodic property can be reflected by the sharpness of the peak. That is, the more periodic the heart sounds become, the sharper the dominant peak will be. In the extreme case, if the heart sounds having ultimate recording time are perfectly periodic, the CFS will have only one nonzero magnitude at the cycle frequency. On the contrary, if the heart rate varies much, the degree of periodic property of the heart sounds reduces greatly; then the peak of the CFS will become flat. In another extreme case, the heart sounds appear randomly; then the CFS will not have any dominant peak. In this sense, the repetition frequency of heart sounds reflects the average frequency of heart rate. So, the repetition frequency can be used as a metric of fetal heart rate. 


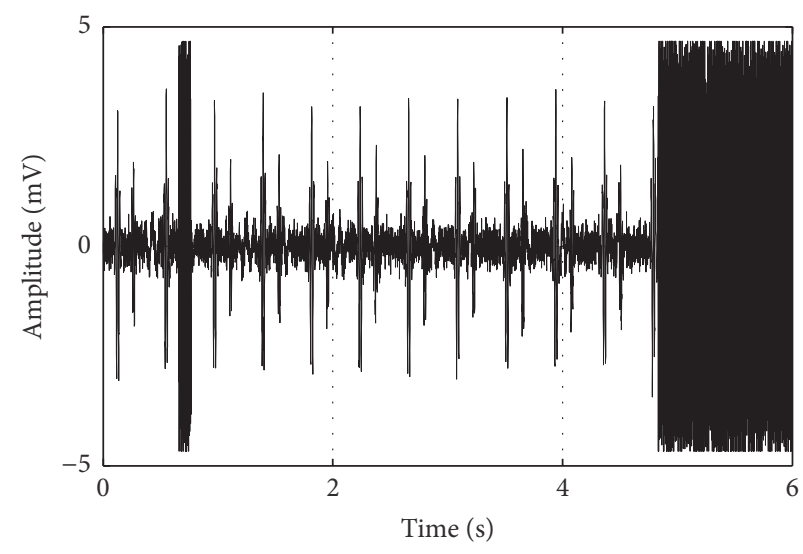

(a)

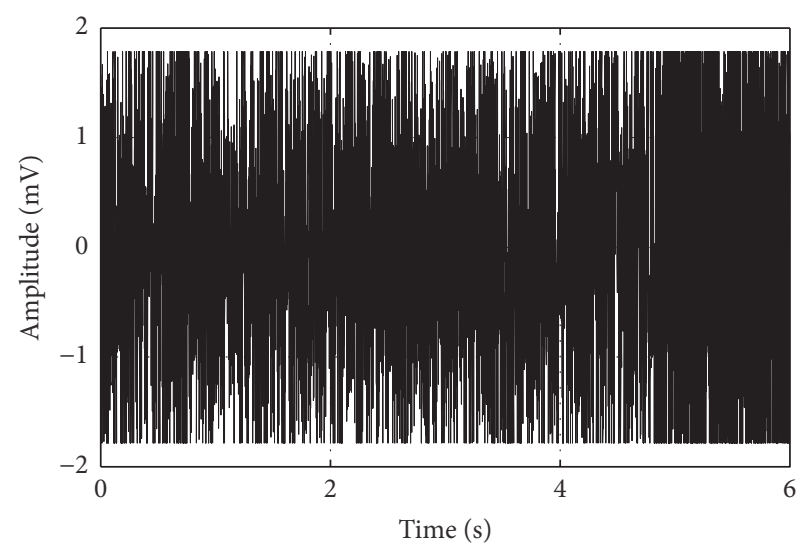

(c)

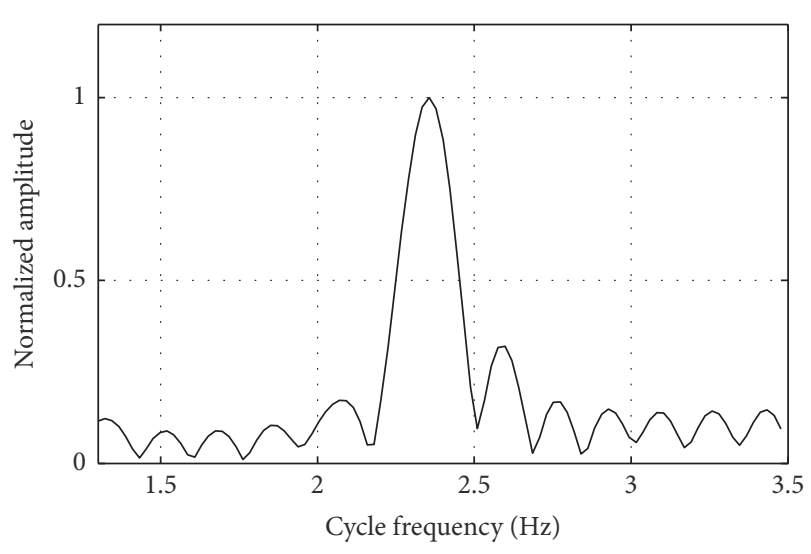

(b)

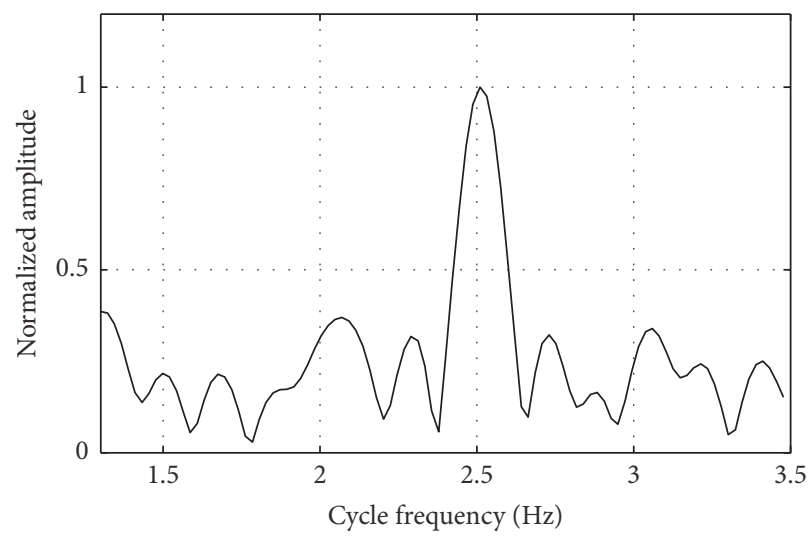

(d)

Figure 1: Fetal heart rate detection for an fPCG signal. (a) A low noise fPCG signal; (b) CFS analysis. Cycle frequency indicated by the dominant peak is $2.35 \mathrm{~Hz}(141 \mathrm{bpm})$. (c) A heavy noise fPCG signal; (d) CFS analysis. Cycle frequency indicated by the dominant peak is $2.51 \mathrm{~Hz}(151 \mathrm{bpm})$.

TABLE 1: Summary of the tests.

\begin{tabular}{lccc}
\hline Record number & SNR $(\mathrm{dB})$ & Heart rate variation $(\mathrm{bpm})$ & Accuracy rate \\
\hline 1 & -4.4 & $108-159$ & $92.3 \%$ \\
2 & -6.6 & $108-159$ & $91.9 \%$ \\
3 & -8.1 & $108-156$ & $90.1 \%$ \\
4 & -10.2 & $108-159$ & $89.1 \%$ \\
5 & -14.8 & $108-168$ & $89.7 \%$ \\
6 & -17.2 & $108-156$ & $90.1 \%$ \\
7 & -20.1 & $108-177$ & $88.5 \%$ \\
8 & -23.8 & $111-153$ & $88.8 \%$ \\
9 & -25.6 & $111-168$ & $82.0 \%$ \\
10 & -26.7 & $108-168$ & $81.0 \%$ \\
\hline
\end{tabular}

Note: the accuracy rate is defined in (9).

2.2. Time-Varying Estimation Using Sliding Window. To track time-varying FHR, a sliding window covering the fPCG signal is used. Therefore, (5) becomes time-dependent:

$$
\gamma_{x}(\alpha, t)=\int_{\infty}^{\infty}\left|S_{x}(\alpha, f, t)\right| d f
$$

where $S_{x}(\alpha, f, t)$ is a cyclic spectral density over a time window $[t-\zeta, t+\zeta]$. So, $\gamma_{x}(\alpha, t)$ can be called time-varying cyclic spectrum. The width of the sliding window is $2 \zeta$. From the repetition feature of heart sound, it can be concluded that the width of the sliding window must be greater than two cardiac cycles to ensure that the fetal heart beats at least 


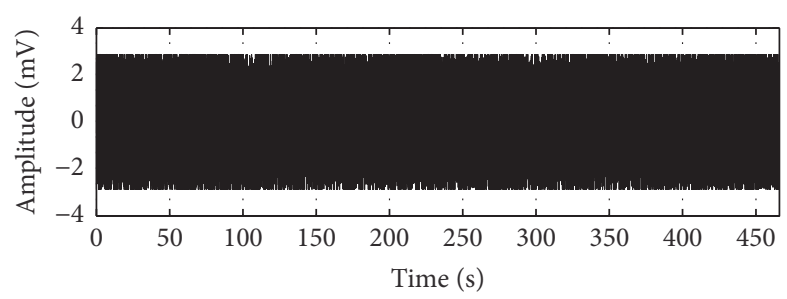

(a)

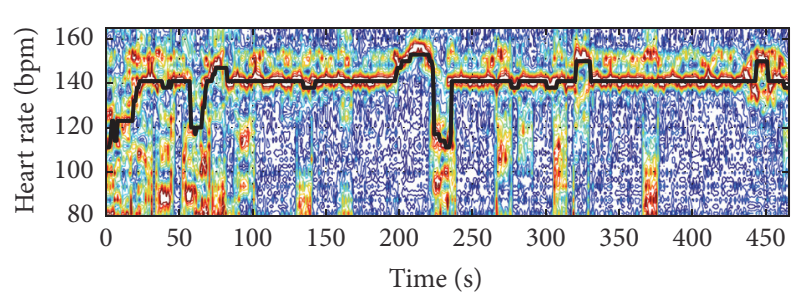

(b)

FIGURE 2: Time-varying fetal heart rate monitoring. (a) An fPCG signal with saturated noise. (b) Detected time-varying fetal heart rate.

two times in the window. Otherwise, there is no repetition feature in the windowed signal. The time-varying FHR can be tracked by searching the peak in $\gamma_{x}(\alpha, t)$ :

$$
\operatorname{Hr}(t)=\arg \left\{\max \left(\gamma_{x}(\alpha, t)\right)\right\} \times 60 .
$$

So, the instantaneous FHR can be detected by the peak location of the time-varying cyclic frequency spectrum.

An fPCG signal is $478 \mathrm{~s}$ in time length, as seen in Figure 2(a). The signal is contaminated by saturated noise. The estimated SNR is $-15.7 \mathrm{~dB}$. A sliding window with width of $8 \mathrm{~s}$ and overlap of $0.1 \mathrm{~s}$ is applied to the signal. The detected timevarying FHR is shown by the black solid line in Figure 2(b). The tracking trajectory accurately reflects the FHR variations. Accelerations and decelerations are clearly observed in the trajectory.

\section{Experiments}

3.1. Time Resolution and Cycle Frequency Resolution. The primary purpose of the sliding window is to limit the extent of the subsequence to be analyzed, so that the cyclic characteristics are approximately constant over the duration of the window. The more rapidly the repetition frequency changes, the shorter the window should be. So, it is reasonable to conclude that as the window length becomes longer, the peak will become sharper; that is, the repetition frequency resolution increases. On the other hand, as the window length decreases, the ability to resolve changes with time increases. Consequently, the choice of window length becomes a tradeoff between repetition frequency resolution and time resolution. With the preknowledge of the change rate of FHR, the window length can be empirically selected as $5-10 \mathrm{~s}$ to obtain an acceptable trade-off.

\subsection{Performance of Fetal Heart Rate Monitoring Using the} Repetition Frequency. To evaluate the proposed method, it is necessary to test the performance under controlled conditions. This is achieved by the simulated fPCG signals $[18,19]$. Each signal lasts $478 \mathrm{~s}$. These signals considered different fetal physiological and pathological conditions and recording situations by simulation technique. The noise considered consists of vibrations created by maternal body organs, fetal movements, unwanted sounds from surrounding environment, and so forth. The SNR ranges from $-4.4 \mathrm{~dB}$ to $-26.7 \mathrm{~dB}$. Visual check by human eyes shows that fetal heart sounds cannot be recognized as the SNR is lower than
$-15 \mathrm{~dB}$. The FHR in each signal is tracked by the proposed method. If the difference between the detected heart rate and the benchmark is within $\pm 5 \mathrm{bpm}$, the detected heart rate is considered accurate. The accuracy rate is defined as

$$
\begin{aligned}
& \text { accuracy rate } \\
& =\frac{\text { number of beats within difference } \leqslant 5 \mathrm{bpm}}{\text { total number of beats }} .
\end{aligned}
$$

A summary of the tests is presented in Table 1. It can be found that the proposed method can accurately track the fetal heart rate even the SNR is lowered to $-26.7 \mathrm{~dB}$, where the heart sounds are totally covered by the noise. The key point why the proposed method is so robust to noise is because it detects the repetition frequency of heart sounds instead of detecting the time difference of heart sounds.

\subsection{Performance Comparison to the Previous Methods. Two} typical previous methods are selected to be compared to the proposed method in this subsection. One is a rule-based method proposed by Kovâcs et al. [16], where the FHR was evaluated by searching S1-S2 pairs. The other method is an advanced method proposed by Várady et al. [14], where the FHR was estimated by the periodicity of heart sound bursts from cross-correlation of signal envelope. Both methods need detection of the fetal heart sound burst. Hence, the performance of the two methods is heavily dependent on the detection accuracy of fetal heart sound burst. Ten simulated fPCG signals with SNR varying from $-4.4 \mathrm{~dB}$ to $-26.7 \mathrm{~dB}$ are used to evaluate the three methods and the results are given in Table 2. It is found that the rule-based method and the advanced method outperform the proposed method in low noise environments. However, both the rule-based and the advanced methods degrade greatly due to the fast increasing of missing rate for sound burst detection with decreasing SNR. The proposed method does not need sound burst detection. It is to estimate the repetition frequency of heart sound, which is less affected by random noise. Hence, the FHR estimation is robust to noise even if the sound burst is destroyed by heavy noise because the repetition feature still remains.

\section{Conclusions}

fPCG is a promising technique to monitor fetal heart rate. However, the performance of previous methods generally 
TABLE 2: Performance comparison.

\begin{tabular}{|c|c|c|c|c|}
\hline Record number & SNR (dB) & The proposed method & $\begin{array}{c}\text { Rule-based method } \\
{[16]}\end{array}$ & $\begin{array}{c}\text { Advanced method } \\
{[14]}\end{array}$ \\
\hline 1 & -4.4 & $92.3 \%$ & $95.4 \%$ & $96.2 \%$ \\
\hline 2 & -6.6 & $91.9 \%$ & $95.4 \%$ & $96.1 \%$ \\
\hline 3 & -8.1 & $90.1 \%$ & $94.1 \%$ & $96.3 \%$ \\
\hline 4 & -10.2 & $89.1 \%$ & $90.3 \%$ & $94.5 \%$ \\
\hline 5 & -14.8 & $89.7 \%$ & $82.6 \%$ & $90.4 \%$ \\
\hline 6 & -17.2 & $90.1 \%$ & $60.8 \%$ & $86.7 \%$ \\
\hline 7 & -20.1 & $88.5 \%$ & - & $82.4 \%$ \\
\hline 8 & -23.8 & $88.8 \%$ & - & $63.6 \%$ \\
\hline 9 & -25.6 & $82.0 \%$ & - & - \\
\hline 10 & -26.7 & $81.0 \%$ & - & - \\
\hline
\end{tabular}

Note: (a) the accuracy rate is defined in (9). (b) "-" means that so many heart sound burst pairs were missed that the accuracy rate was very low.

depends on the detection rate of heart sound bursts from an fPCG recording. The authors find that the fetal heart rate can be evaluated by the repetition frequency of heart sounds, which can be extracted from peaks in the cyclic frequency spectrum without sound burst detection and denoising. The feature of repetition can remain even if the SNR is lowered to $-26.7 \mathrm{~dB}$. As the SNR is lower than $-15 \mathrm{~dB}$, the proposed method outperforms the two typical previous methods.

\section{Competing Interests}

The authors declare that there are no competing interests regarding the publication of this paper.

\section{Acknowledgments}

This work was supported in part by the National Natural Science Foundation of China (Grant nos. 61471081 and 61601081) and Fundamental Research Funds for the Central Universities (Grant nos. DUT15QY60, DUT16QY13, DC201501056, and DCPY2016008).

\section{References}

[1] M. Romano, L. Iuppariello, A. M. Ponsiglione, G. Improta, P. Bifulco, and M. Cesarelli, "Frequency and time domain analysis of foetal heart rate variability with traditional indexes: a critical survey," Computational and Mathematical Methods in Medicine, vol. 2016, Article ID 9585431, 12 pages, 2016.

[2] R. T. Wakai, "Assessment of fetal neurodevelopment via fetal magnetocardiography," Experimental Neurology, vol. 190, S1, pp. 65-71, 2004.

[3] R. M. Grivell, Z. Alfirevic, G. M. Gyte, and D. Devane, "Antenatal cardiotocography for fetal assessment," Cochrane Database of Systematic Reviews, no. 1, Article ID CD007863, 2010.

[4] H. Cao, D. E. Lake, J. E. Ferguson, C. A. Chisholm, M. P. Griffin, and J. R. Moorman, "Toward quantitative fetal heart rate monitoring," IEEE Transactions on Biomedical Engineering, vol. 53, no. 1, pp. 111-118, 2006.

[5] M. Romano, M. Bracale, M. Cesarelli et al., "Antepartum cardiotocography: a study of fetal reactivity in frequency domain,"
Computers in Biology and Medicine, vol. 36, no. 6, pp. 619-633, 2006.

[6] M. Anisha, S. S. Kumar, and M. Benisha, "Methodological survey on fetal ECG extraction," Journal of Health \& Medical Informatics, vol. 5, article 469, 2014.

[7] F. Kovács, C. Horváth, Á. T. Balogh, and G. Hosszú, "Fetal phonocardiography-past and future possibilities," Computer Methods and Programs in Biomedicine, vol. 104, no. 1, pp. 19-25, 2011.

[8] F. Kovács, N. Kersner, K. Kádár, and G. Hosszú, “Computer method for perinatal screening of cardiac murmur using fetal phonocardiography," Computers in Biology and Medicine, vol. 39, no. 12, pp. 1130-1136, 2009.

[9] F. Kovács, C. Horváth, Á. T. Balogh, and G. Hosszú, “Extended noninvasive fetal monitoring by detailed analysis of data measured with phonocardiography," IEEE Transactions on Biomedical Engineering, vol. 58, no. 1, pp. 64-70, 2011.

[10] V. S. Chourasia, A. K. Tiwari, and R. Gangopadhyay, “Timefrequency characterization of fetal phonocardiographic signals using wavelet scalogram," Journal of Mechanics in Medicine and Biology, vol. 11, no. 2, pp. 391-406, 2011.

[11] M. Moghavvemi, B. H. Tan, and S. Y. Tan, "A non-invasive PCbased measurement of fetal phonocardiography," Sensors and Actuators A: Physical, vol. 107, no. 1, pp. 96-103, 2003.

[12] V. S. Chourasia, A. K. Tiwari, and R. Gangopadhyay, "A novel approach for phonocardiographic signals processing to make possible fetal heart rate evaluations," Digital Signal Processing: A Review Journal, vol. 30, pp. 165-183, 2014.

[13] M. Ruffo, M. Cesarelli, M. Romano, P. Bifulco, and A. Fratini, "An algorithm for FHR estimation from foetal phonocardiographic signals," Biomedical Signal Processing and Control, vol. 5, no. 2, pp. 131-141, 2010.

[14] P. Várady, L. Wildt, Z. Benyó, and A. Hein, "An advanced method in fetal phonocardiography," Computer Methods and Programs in Biomedicine, vol. 71, no. 3, pp. 283-296, 2003.

[15] J. Chen, K. Phua, Y. Song, and L. Shue, "A portable phonocardiographic fetal heart rate monitor," in Proceedings of the IEEE International Symposium on Circuits and Systems (ISCAS '06), May 2006.

[16] F. Kovâcs, M. Török, and I. Habermajer, "A rule-based phonocardiographic method for long-term fetal heart rate monitoring," IEEE Transactions on Biomedical Engineering, vol. 47, no. 1, pp. 124-130, 2000. 
[17] S. Vaisman, S. Yaniv Salem, G. Holcberg, and A. B. Geva, "Passive fetal monitoring by adaptive wavelet denoising method," Computers in Biology and Medicine, vol. 42, no. 2, pp. 171-179, 2012.

[18] M. Cesarelli, M. Ruffo, M. Romano, and P. Bifulco, "Simulation of foetal phonocardiographic recordings for testing of FHR extraction algorithms," Computer Methods and Programs in Biomedicine, vol. 107, no. 3, pp. 513-523, 2012.

[19] Simulated fetal PCGs, July 2015, http://www.physionet.org/physiobank/database/simfpcgdb/. 


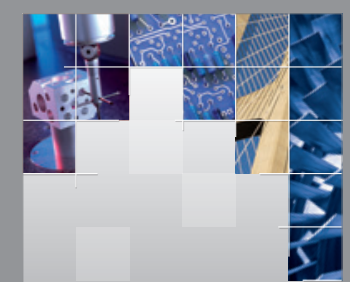

\section{Enfincering}
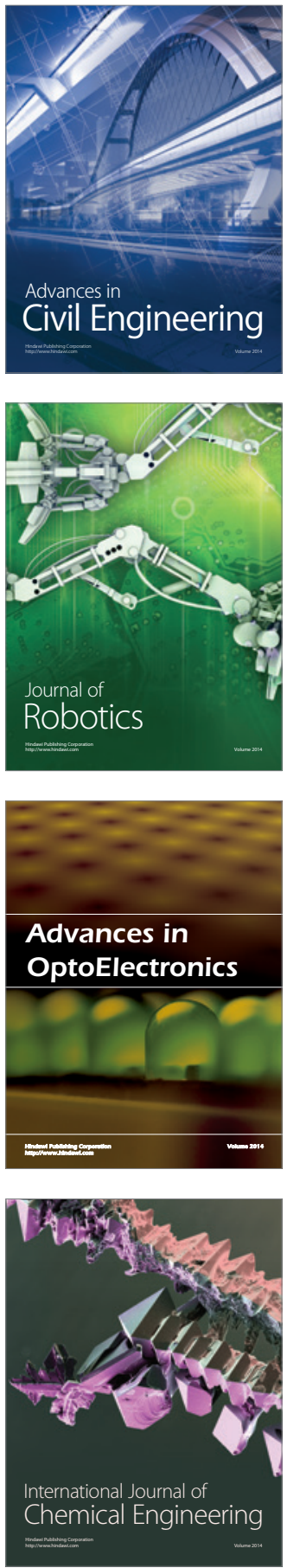

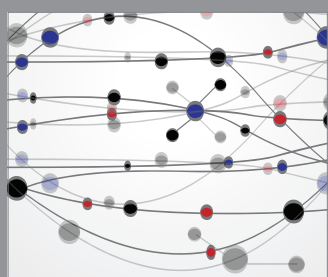

The Scientific World Journal

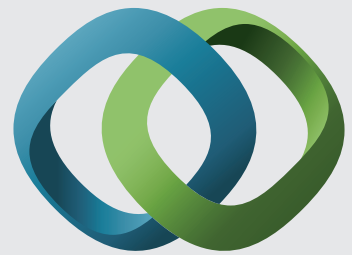

\section{Hindawi}

Submit your manuscripts at

http://www.hindawi.com
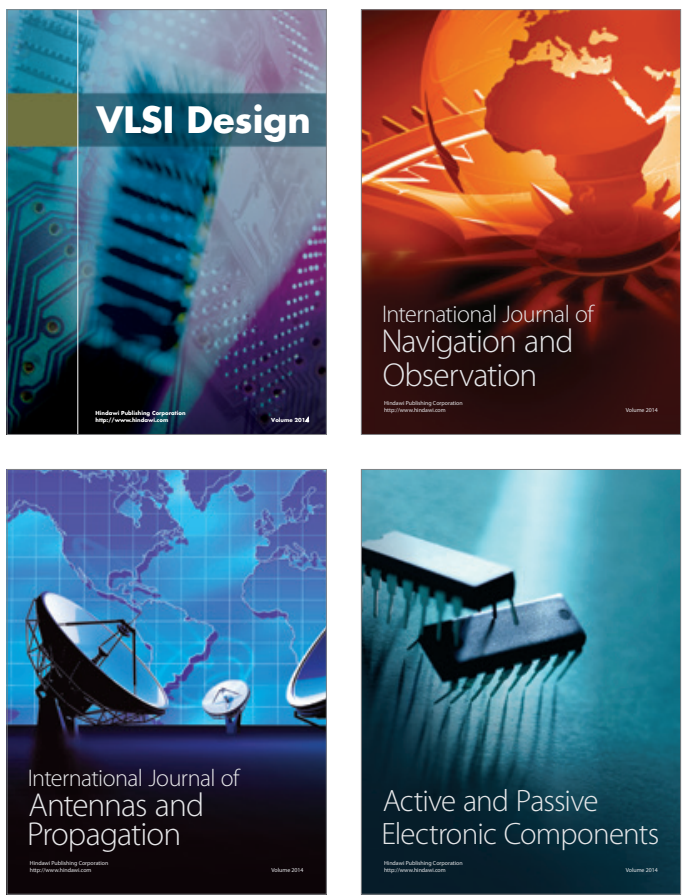
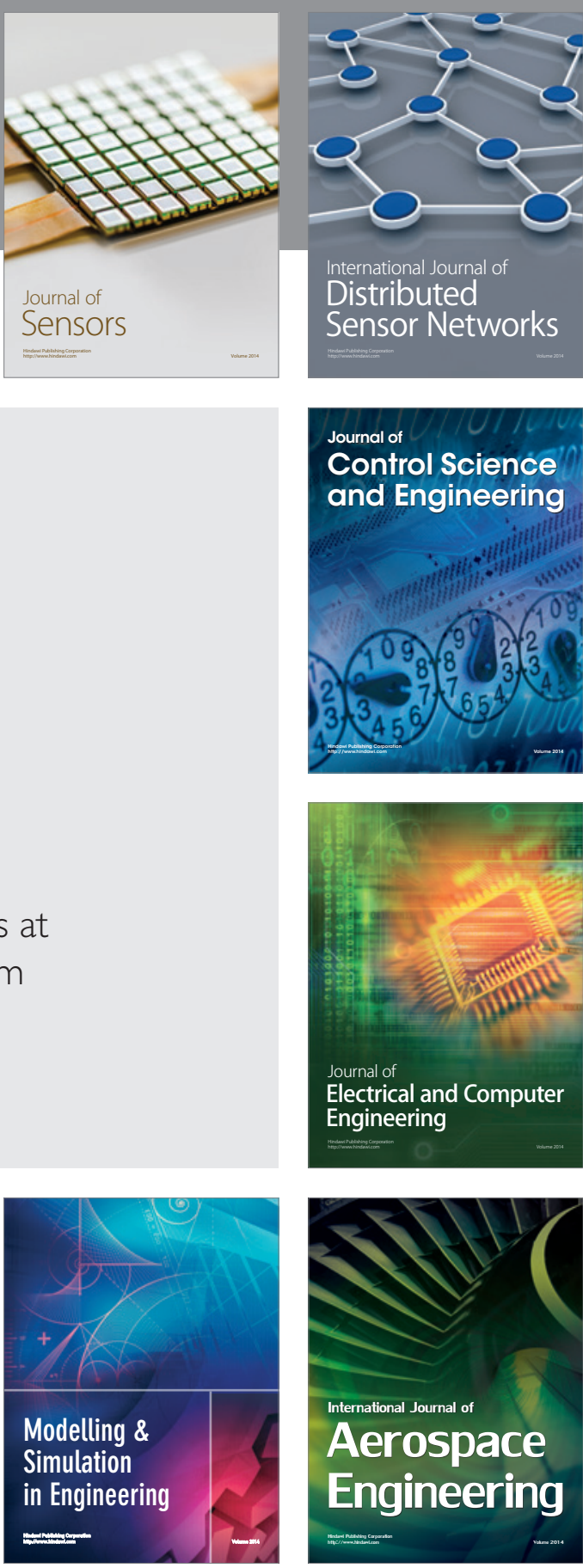

International Journal of

Distributed

Sensor Networks

Journal of

Control Science

and Engineering
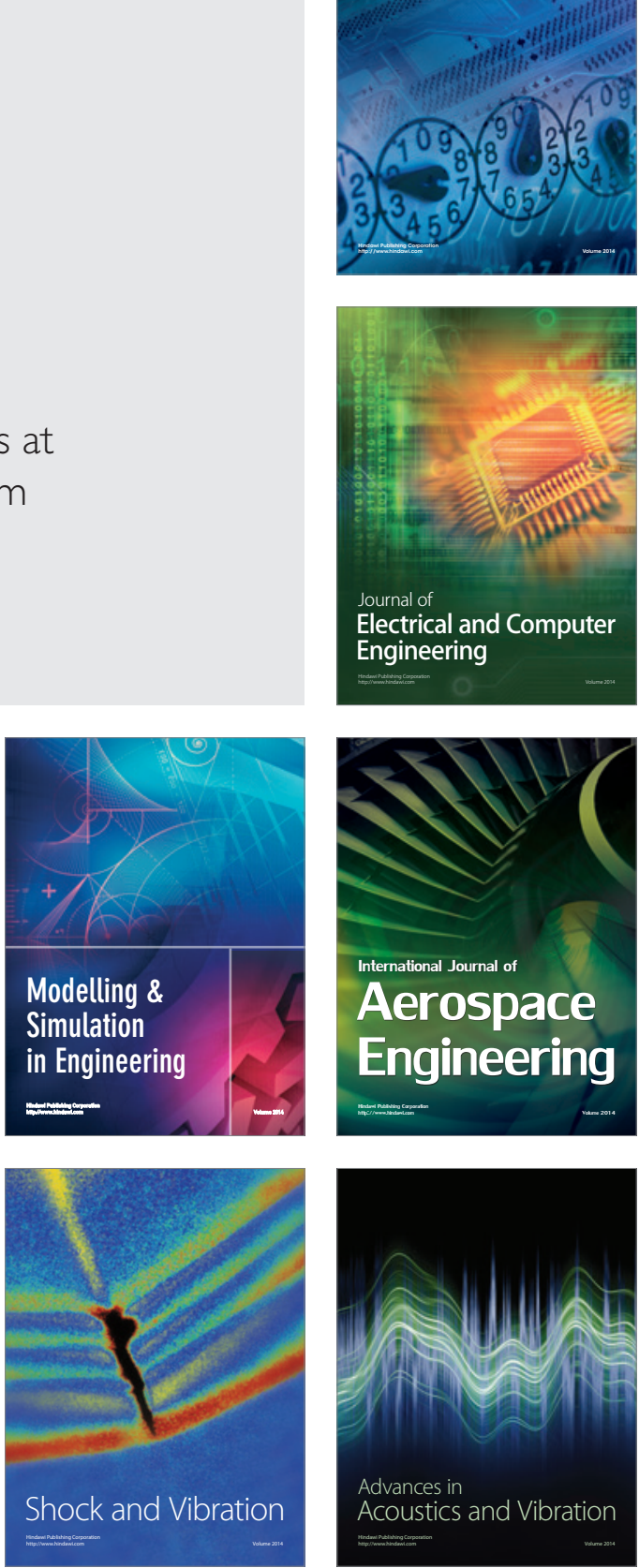